\title{
WMAP Observatory Thermal Design and On-Orbit Thermal Performance
}

\author{
Stuart D. Glazer, Kimberly D. Brown and Theodore J. Michalek \\ NASA/Goddard Space Flight Center \\ Walter C. Ancarrow \\ WCA Engineering, Inc.
}

\begin{abstract}
The Wilkinson Microwave Anisotropy Probe (WMAP) observatory, launched June 30,2001 , is designed to measure the cosmic microwave background radiation with unprecedented precision and accuracy while orbiting the second Lagrange point (L2). The instrument cold stage must be cooled passively to $<95 \mathrm{~K}$, and systematic thermal variations in selected instrument components controlled to less than $0.5 \mathrm{mK}$ (rms) per spin period. This paper describes the thermal design and testing of the WMAP spacecraft and instrument. Flight thermal data for key spacecraft and instrument components are presented from launch through the first year of mission operations. Effects of solar flux variation due to the Earth's elliptical orbit about the sun, surface thermo-optical property degradations, and solar flares on instrument thermal stability are discussed.
\end{abstract}

\section{INTRODUCTION}

The Wilkinson Microwave Anisotropy Probe (WMAP) observatory, launched June 30,2001 , is designed to measure the cosmic microwave background (CMB) radiation over the full sky with unprecedented precision and accuracy. The Medium Explorer (MIDEX) class observatory was designed, built, and tested principally by engineers and scientists at Princeton University and the NASA Goddard Space Flight Center (GSFC). Dr. Charles Bennett of the NASA Goddard Space Flight Center is the Principal Investigator. Additional scientists from NASAVGSFC, Brown University, UCLA, the University of Chicago, and the University of British Columbia complete the makeup of the science team. Originally named MAP, the mission was renamed in 2003 in memory of Dr. David Wilkinson, a Professor of Physics at Princeton University and pioneer in the field of $\mathrm{CMB}$ research, who died in 2002. Dr. Wilkinson was a mission Co-Investigator.

The WMAP mission was envisioned as a follow-on to the Differential Microwave Radiometer (DMR) experiment flown on NASA's Cosmic Background Explorer (COBE) mission launched nearly a decade earlier. The WMAP instrument measures the variation, or anisotropy, in

temperature over the entire sky with improved spatial resolution and sensitivity, compared with the DMR instrument.

The extraordinarily subtle directional variation in background temperature over the full sky (on the order of microKelvins), reflects conditions existing approximately 380,000 years after the "Big Bang", 13.7 billion years ago. Careful measurement of the anisotropy in sky temperature of the CMB permits quantitative evaluation of many key cosmological parameters. It has already helped to shed light on important questions raised by current cosmological theories, including:

- Is the universe dominated by exotic "dark matter"?

- How and when did the first galaxies form?

- Was there a period of inflationary expansion?

- Will the universe continue to expand forever, or will it collapse upon itself?

- Is the expansion of the universe accelerating or decelerating?

- What is the shape of the universe?

To answer these questions, WMAP measures the anisotropy of the $\mathrm{CMB}$ over the full sky with a sensitivity of $20 \mu \mathrm{K}$ and an angular resolution of at least 0.3 degrees. in contrast to DMR's 6 differential radiometers measuring microwave energy in 3 wavelengths and 7.0 degree spatial resolution, WMAP uses 10 differencing radiometers to measure the microwave energy in 5 wavelengths from 22 to $90 \mathrm{GHz}$ with a spatial resolution of 0.3 degrees. WMAP also employs far more sensitive state-of-the-art High Energy Mobility Transistor (HEMT) amplifiers, operates at a lower temperature $(<95 \mathrm{~K}$ vs approximately $145 \mathrm{~K}$ ), and is much more thermally stable. This is due in part to its orbit about the second Lagrange point, nearly $1.5 \times 10^{6} \mathrm{~km}$ from the Earth, and the satellite design, in which the instrument cold stage is constantly shadowed from solar flux, and nearly always shadowed from Earth emitted infrared energy. 


\section{MISSION OVERVIEW}

The WMAP Observatory was launched aboard a Delta rocket from Cape Canaveral, Florida, on June 30, 2001 into a roughly circular orbit about the Earth. Before the end of the first orbit, the Delta's third stage engine fired, putting WMAP into a highly elliptical orbit designed to allow the observatory to make use of lunar gravity assist to reorient it towards L2. A total of three "phasing loops", each lasting approximately 9 days, were required prior to lunar swingby on July 30,2001 . WMAP's solar panels/sunshades were deployed immediately following third stage engine bum and jettison. It maintained observing mode attitude, spin, and orientation relative to the sun from that point on, except during an initial checkout period, brief orbital adjustments during the phasing loops (perigee), and lunar swingby (perilune). The maneuvers exposed normally shadowed parts of the observatory to direct solar energy, and resulted in rapid temperature excursions during each exposure period. These brief periods of solar exposure demanded extensive transient thermal analysis during the observatory design, and had an significant influence on observatory materials and coatings selection. Although science data was collected throughout the entire cruise period, the first of several sky maps completed to date was officially begun upon L2 orbit insertion, which was officially declared in early October 2001. The original mission design life was 27 months, allowing three months for cruise to $L 2$, plus 24 months gathering science data at $L 2$, sufficient for four complete sky coverages. The mission has since been extended an additional 24 months, allowing for four additional sky coverages. This is made possible in part by the use of passive instrument thermal control, plus minimal fuel requirements for the three brief trajectory correction maneuvers (TCM) performed at L2 annually. Figure 1 illustrates the trajectory to and orbit about $L 2$.

Top View
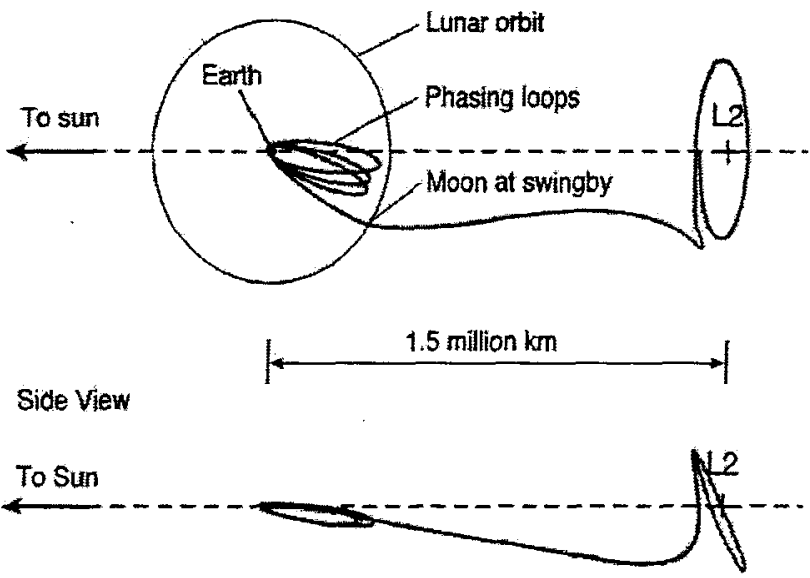

Figure 1. WMAP Trajectory to L2

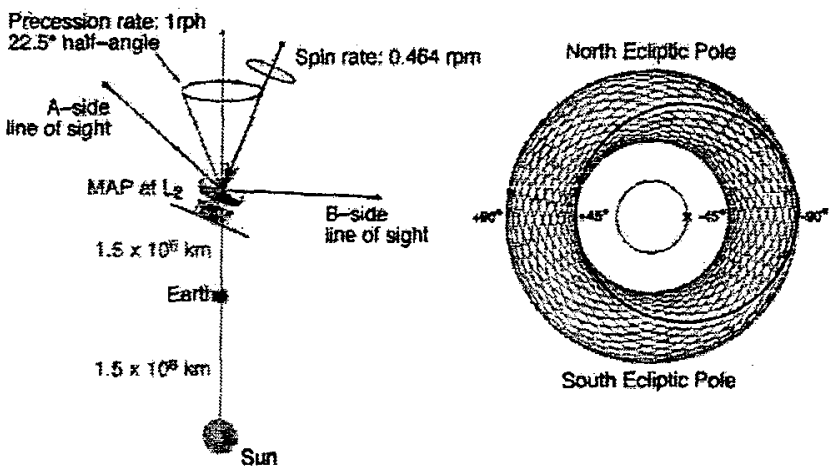

Figure 2. WMAP Scan Pattern

The clever observational scan strategy is illustrated in Figure 2. It requires the observatory to spin about its $Z$ axis approximately once every 2.2 minutes, while maintaining a constant angle between the sun and the spin axis of 22.5 degrees. The $Z$ axis precesses about the WMAP sunline once per hour. Approximately $30 \%$ of the sky is viewed during each precession period. Coverage of the full sky is completed every six months, as the orbit of the Earth (and the L2 point) reaches the opposite sides of the sun, while the observatory maintains its orientation relative to the sun. Each point on the sky is observed thousands of times in the course of the mission, enhancing measurement sensitivity.

\section{KEY THERMAL REQUIREMENTS}

The mission was designed to keep pixel to pixel variance due to the systematic effects of the instrument to $5 \%$ of the random noise. The total systematic error was therefore specified as $<5 \mu \mathrm{K}$ rms per sky map pixel, which translates to a key thermal stability requirement for selected microwave components of $<0.5 \mathrm{mK}$ rms per spin period. This tight requirement was necessary because instrument offset signals must be differentiated from the true sky signals as the differencing radiometers view the same point in the sky over a spin period. This in turn -drives the thermal requirement over à spin pēriō because spin modulated thermal variations cannot be easily distinguished from true sky signals. Thermal stability requirements over longer terms were not necessary.

The stringent thermal control requirements of the instrument dictated thermal control techniques and methodologies to be used in the thermal design of the entire observatory. Additional thermal requirements for the instrument are listed in Table A1 (in the appendix).

\section{SPACECRAFT AND INSTRUMENT THERMAL DESIGN}

Key elements of the observatory design are shown in Figure 3. The stringent thermal requirements listed in A1 had to be met despite WMAP's designation as a MIDEX mission, which imposed severe limitations on mass, power, schedule, and cost. The principal spacecraft subsystems consist of the following: power, 
command and data handing, communications, propulsion (for orbit and station keeping maneuvers), and attitude control. "Room temperature", or "warm" spacecraft and instrument electronics boxes are mounted externally (without enclosed bays) onto a sixsided M46J carbon

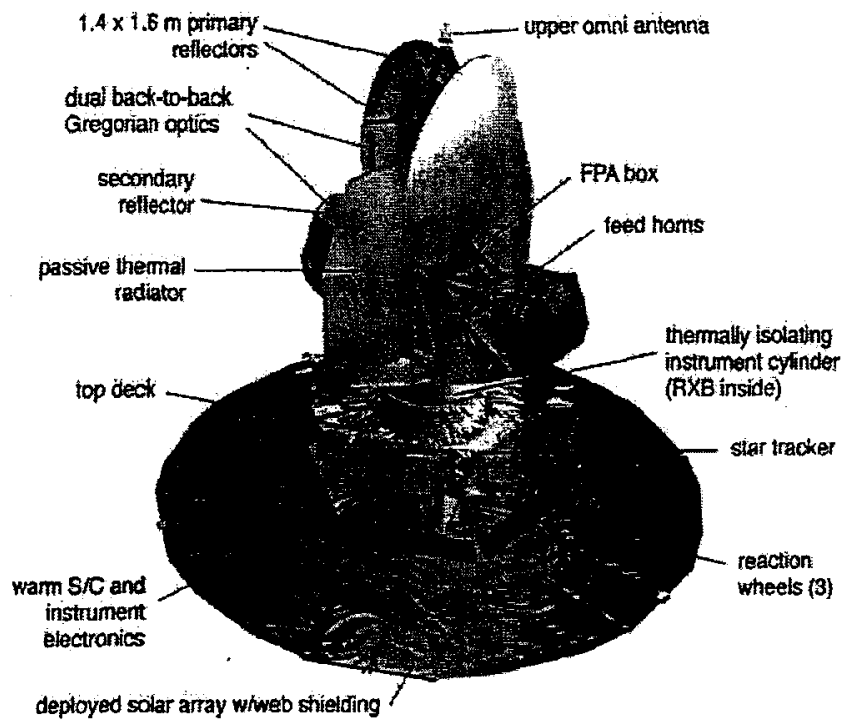

Figure 3. WMAP Observatory

composite bus structure approximately $76.2 \mathrm{~cm}$ tall. Since the structure is a relatively poor heat conductor, the spacecraft thermal design is based on local heat rejection to space directly from individual components. This is augmented on selected boxes and propulsion lines with operational heaters controlled by on-board software. The open geometry was adopted to reduce mass and to help radiatively isolate spacecraft components from the warm instrument electronics boxes to achieve temperature stability over a spin period, but it required extensive and complex blanketing over the decks and around the hexagonal bus structure. The propulsion system tank is located inside the hexagonal bus, while most of the lines to the thrusters run under the $\mathrm{MLI}$ on bottom and top decks.

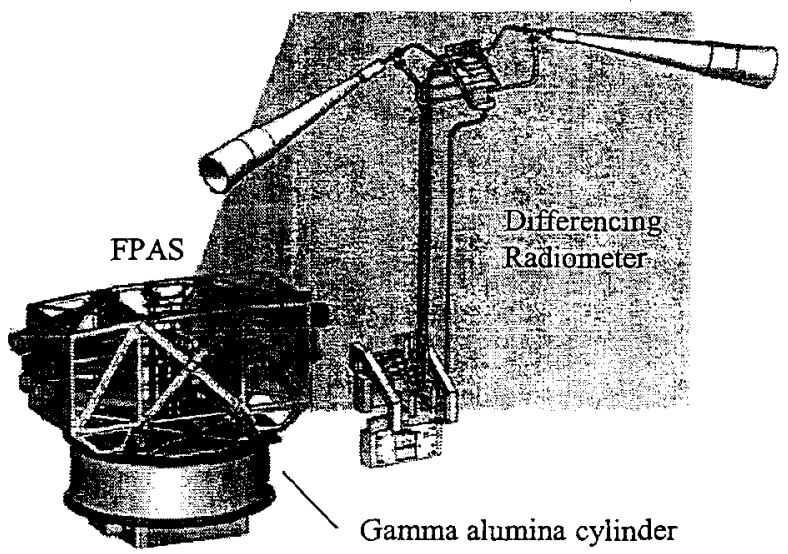

Figure 4. Microwave Instrument, with detail of a single Differencing Radiometer
The 95K cold stage of the instrument is comprised of the carbon composite primary/secondary microwave reflectors, passive aluminum radiator, and support structure, known collectively as the Thermal Reflector System (TRS), plus cold microwave amplifiers, components, waveguides, and feedhorns mounted in the Focal Plane Assembly Structure (FPAS). Further details of the microwave section of the instrument are seen in Figure 4. The cold stage is structurally mounted to but thermally isolated from the top deck/warm bus region primarily by a gamma-alumina cylinder approximately $0.305 \mathrm{~cm}$ thick and $31.5 \mathrm{~cm}$ in length and coated with highly emissive A276 white paint. Both the TRS and gamma alumina cylinder were exposed to solar loading during the launch and early orbit phase, requiring thorough analysis and testing. Microwave signals entering the cold feedhorns are first amplified by cold amplifiers, and then transmitted to a set of warm (room temperature) amplifiers in the Receiver Box (RXB) through 40 copper waveguides. A stainiess steei section $20.32 \mathrm{~cm}$ long, $0.0254 \mathrm{~cm}$ thick is incorporated into each copper waveguide to provide conductive thermal isolation between warm and cold instrument stages. Additional radiative isolation between the warm $\mathrm{RXB}$ and the instrument cold stage is attained by complex MLI blankets at the interface. The warm and cold microwave sections also had to be isolated to prevent any microwave leaks in one section from contaminating the other. This initially caused substantial parasitic heat leaks to the cold stage when aluminum tape was first used to close out the interface MLI around each stainless steel waveguide section. The heat leak was greatly reduced when the waveguide/MLI interfaces were subsequently closed out with a single layer of VDA-2 kapton, $0.0127 \mathrm{~mm}$ thick. Thermal control of the $R X B$, which is surrounded by the gamma-alumina cylinder, is accomplished by tailoring copper heat strap connections to a small radiator mounted external to the lower cylinder mount flange. The RXB had to be rigidly mounted to the cold FPAS to support the microwave components. Thermal isolation across this interface was accomplished by mounting the FPAS on a truss consisting of 8 thin walled gamma-alumina tubes, with the majority of the heat leak across four $6.9 \mathrm{~cm}$ long, $0.0762 \mathrm{~cm}$ thick tubes. The nearly 500 electrical wires crossing the warm/cold interface were approximately 1 meter long, and were composed of 28 gauge stainless steel.

Electrical power dissipated in the cold amplifiers, plus parasitic heat, is rejected principally from two symmetrical radiator panels, each employing two aluminum 1100 facesheets of variable thickness over a $5.08 \mathrm{~cm}$ thick aluminum honeycomb core. These aluminum radiator panels reject heat to space from both sides of each panel, providing a total of $5.45 \mathrm{~m}^{2}$ radiating area. Each of the radiator panel facesheets are thermally coupled to the cold amplifiers via custom fabricated heat straps consisting of stacked layers of $0.0254 \mathrm{~cm}$ thick aluminum 1100. This alloy was selected for the radiator panel facesheets and the heat straps because its high purity results in an increasing 
thermal conductivity as the temperature drops from $293 \mathrm{~K}$, reaching a peak value (measured) of approximately $3.5 \mathrm{~W} / \mathrm{cm}-\mathrm{K}$ at $90 \mathrm{~K}$. Radiator panel thickness and strap cross sectional area were originally designed to maintain no larger than a $12 \mathrm{~K}$ temperature drop from the cold amplifiers to the low temperature end of the radiator. Actual performance in flight shows a temperature drop of approximately 8-9K. Instrument cold stage heat rejection to space is thus completely passive. The two room temperature instrument electronics boxes requiring extreme thermal stability are the Power Distribution Unit (PDU), and the Analog Electronics Unit/Digital Electronics Unit (AEU/DEU), both of which are mounted on the spacecraft hub.

Table A2 summarizes how the heat loads to the instrument cold stage grew over time. The thermal margin calculated during the proposal period was nearly $100 \%$, which would have permitted instrument cold amplifiers to reach approximately 85K. Early growth in heat loads, particularly due to state-of-the-art HEMT amplifier dissipation growth, wirecounts, and actual workmanship of complex interfaces resulted in final thermal margin of approximately $40 \%$ (in flight). Thermal margin is here defined as the allowable growth in heat load which can still meet the instrument cryogenic temperature requirement with the as-built thermal hardware.

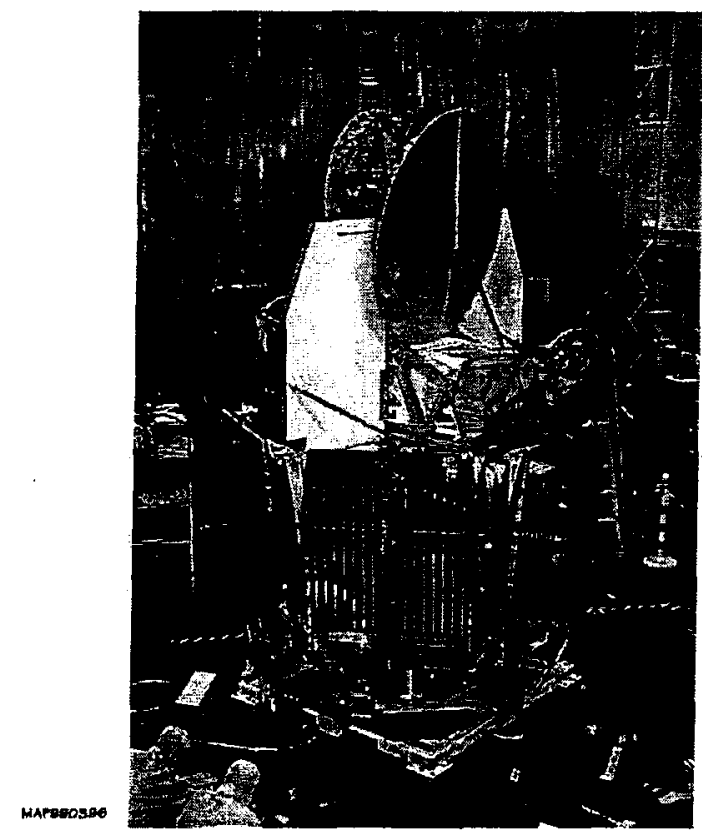

Figure 5. WMAP Observatory, Stowed Configuration

Solar arrays and sunshades are clearly seen in Figure 5, which is a photograph of the observatory in the stowed configuration. The backs of the WMAP solar arrays are blanketed with 18 layer MLI blankets to attenuate heat transfer to the instrument cold stage. This differs from most spacecraft with non-body mounted panels, which allow the panels to reject heat to space from both sides, maintaining panel temperatures generally in the 70-80C temperature range. The silicon solar cells on the WMAP panels were kept in this range by covering nearly $60 \%$ of the panel front surface with Optical Solar Reflectors (OSR's). The arrays are electrically arranged into two "strings" of cells per panel. The nominally $400 \mathrm{~W}$ observatory does not at present require the power from all strings, allowing excess electrical power to be left on selected panels. The sun-facing surface of the 12 layer MLI forming the web sunshades (between the panels) is silver teflon. Note that the sunshades, which are nearly taut in the deployed configuration, are gently folded near the spacecraft when stowed.

The solar arrays and web sunshades, which deployed shortly after launch at the S/C bottom deck, or sunfacing end, were designed to prevent exposure of the instrument cold stage to direct solar flux during science observation. Their size was defined by the size of the TRS, plus the constant angle between the sun and the observatory spin axis of 22.5 degrees while observing. This design aiso results in shadowing of the warm bus region, which further enhances the effectiveness of box radiators (no environmental thermal loads), and their thermal stability (no variation in environmental loads as the S/C spins). The solar arrays and sunshades prevent exposure of the TRS to solar flux with nearly 5 degrees of margin, or over an angle between the sun and the spin axis of $+/-27.5$ degrees. The angle between the Earth and the WMAP spin axis can seasonally reach nearly +/-32 degrees, exposing a very small portion of the cold stage to emitted Earth infrared energy. However, at the nominal Earth-WMAP distance of approximately $1.5 \times 10^{6} \mathrm{~km}$, even the maximum possible exposure to Earth IR flux results in an added spinaveraged absorbed parasitic power of the order of 10 $\mathrm{mW}$ or less, which is insignificant compared to the approximately 11 watts of nominal instrument cold stage thermal+parasitic heat loads. Variations in instrument temperature over the spin period have not exceeded requirements.

Several significant challenges were surmounted in the development of the thermal design. The stability requirements of the instrument dictated that any heaters used on the entire observatory be either fully on or fully off, with no duty cycling. For this reason, replacement heater power is supplied to the transponder when it is not transmitting (after launch, the transponder was set to transmit continuously, further reducing daily observatory temperature variations at the milikelvin level). The extremely cold sink temperature made the design more sensitive to the MLI properties than for a low Earth orbiting spacecraft, particularly for the thrusters and propulsion lines. The sizing of some radiators and the propulsion system heaters could thus not be finalized until the flight system was tested in a thermal vacuum environment. This dictated a unique testing strategy.

Most outer surfaces were required to be electrically conductive and grounded. The dominant MLI outer layer used was vacuum deposited aluminum/kapton/indium tin oxide (VDA/kapton/ITO), ITO side facing out. The fragility of the coating imposed special handling 
procedures during integration and test. Nearly all radiator surfaces on the entire observatory were coated with NS43G, an electrically conductive silicate-based white paint with good emissive properties from room temperature to $80 \mathrm{~K}$. NS43G paint emittance versus temperature is shown in Figure 6 , as measured by transient test techniques at NASA/GSFC. The white paint was used because of brief exposure to the sun during orbital maneuvers. While flight tested and chemically designed to adhere to aluminum, this paint occasionally chipped when handled.

Extensive thermal analysis was performed to guarantee that the design of the TRS remained below their maximum temperature limits during all phases of the mission, particularly during the brief solar exposures during the initial orbit and later trajectory correction maneuvers. As a result, the primary and secondary reflectors, composed of two XN-70 carbon-composite facesheets over a lightweight honeycomb Korex core, were aluminized, but were coated with silicon oxide (SiOx) of carefully controlled thickness, to moderate the solar absorptance/emittance ratios when the reflectors were briefly exposed to the sun during those maneuvers, to prevent overheating.

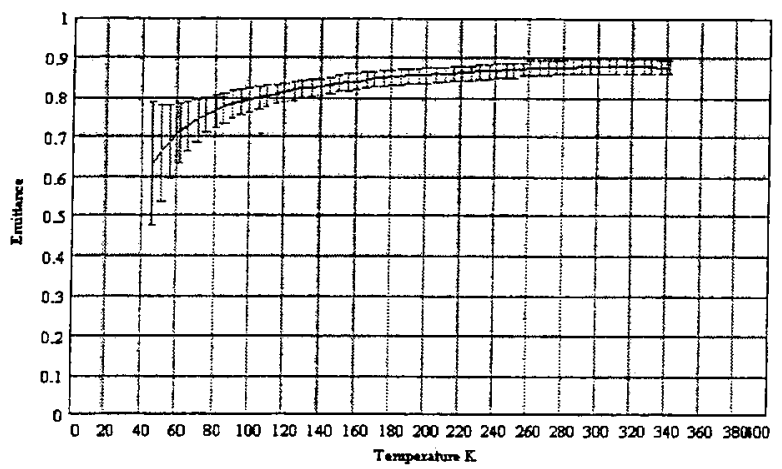

Figure 6. Emittance of NS43G Paint versus Temperature

The importance of spin periodic thermal stability of the microwave components has aiready been discussed. Extensive thermal analysis was performed to calculate the transient response of the microwave system to thermal disturbances occurring at the spin period during science observation. Potential perturbations included power variation in the three reaction wheel electronics boxes (which individually would cycle sinusoidally at the spin period), and worst case sagging in the MLI sunshades (resulting in non-flat surfaces exposed to solar flux). The transient TSS/SINDA instrument models were run for approximately 1000 spin periods, with the amplitudes of the forcing functions magnified until confidence was gained that microwave component stability requirements would likely be achieved passively.

\section{TESTING}

The WMAP instrument underwent extensive thermal testing at multiple stages throughout the design and fabrication phases. Table A3 summarizes thermal vacuum, thermal balance, performance, and qualification tests, configuration, purpose, and key test results. This table does not list tests performed at the component or subassembly level. Of particular importance were the Structural Thermal Mockup (STM) test, and the ColdVibe-Cold (CVC) instrument performance tests. The STM testing provided an early opportunity to thoroughly examine the instrument cold stage thermal design. Analysis of test results pointed to heat leakages, and the planned multi-part testing allowed time to build and test improved designs and implementations. Thermal testing performed during the CVC tests highlighted the need for improved RXB thermal control. The improved thermal control methodology was devised, fabricated, and installed while the test was interrupted as faulty flight amplifiers were being repaired. This highlights that some of the most valuable data gained from these tests were unexpected.

The thermal success of the mission may be attributed in large measure to the extensive test program initiated early in the design/fabrication phases. It allowed time to understand and correct potential problems in instrument thermal design and workmanship in time for the flight unit development.

\section{Flight Thermal Data}

The WMAP instrument incorporated a specially designed system to measure selected instrument temperatures using Platinum Resistance Thermometers (PRT's) with a precision of $0.5 \mathrm{mK}$, at a peak rate of approximately 6 times per spin period (every 22 seconds), for both the warm and cryogenic instrument stages. Its actual performance in flight indicates a precision closer to $0.1 \mathrm{mK}$. This was necessary because the Observatory's Command and Data Handling subsystem, which measured spacecraft temperatures using thermistors, had neither the thermal range nor the precision to verify that instrument thermal stability met the mission requirements. Results from both sensor types are shown below. All of the following figures display temperatures compiled at 20 minute intervals, except for Figure 11, which utilizes all sampled data (at approximately 22 second intervals).

Figures 7 and 8 show selected spacecraft component and propulsion line temperatures from launch on June 30,2001 , through January 6, 2003. Figure 9 illustrates selected temperatures on the warm instrument components, while Figure 10 shows temperatures of a representative cold stage microwave magic tee element ( 1 of 5 instrumented out of 20 total, and physically near the amplifiers), a feedhorn, and the top of the " $A$ " primary reflector over the same period. Flight temperatures seen in Figures 7-10 met all thermal requirements, as shown in Table A1. Further, thermal predictions made with models correlated after Observatory thermal vacuum/thermal balance tests, for the instrument warm boxes, and warm and cold microwave components were highly accurate. The 
AEU/DEU, PDU, and RXB box were predicted to lie in the ranges of $18.7 \mathrm{C}-32.3 \mathrm{C}, 16.4 \mathrm{C}-30.8 \mathrm{C}, 6.5 \mathrm{C}-17.5 \mathrm{C}$, respectively, throughout the mission, given the remaining uncertainties, such as MLI effective emittance. Cold stage microwave components were predicted to lie in the range of $87.9 \mathrm{~K}-93.2 \mathrm{~K}$. Actual flight temperatures of the primary reflectors at the top were approximately $7 \mathrm{~K}$ higher than predicted by the model, due in part to the complex low thermal conductivity composite structure, and low heat loads leaking into the reflectors. This had no adverse impact on the mission.

Figures 11 and 12 illustrate the thermal stability of all 5 of the instrumented cold magic tees, and top of the " $A$ " primary reflector while observing. The sharp discontinuity on February 27, 2002 was the result of a battery cell failure, and the subsequent resetting of the battery charge rate. Even a relatively minor change in a spacecraft temperature is observed in the well isolated instrument cold stage at the milliKelvin level. Figures 9 and 10 illustrate how cold stage temperatures vary sinusoidally over a year, and display a slight upward bias. The sinusoidal component results from the annual variation in sun-Earth (and sun-L2) distance of $+/$ $1.637 \%$ (eccentricity of the Earth's orbit about the sun). This results in a nominal annual variation of approximately $+/-3.4 \%$ in incident solar flux at the spacecraft solar panels/sunshades, which affects temperatures over the entire observatory. The upward bias results from the continual degradation of solar absorptances at the spacecraft sun-side, and may be modeled to first order as an exponential function. The annual sinusoidal temperature variation of the instrument cold stage components is of the order of +1 $0.2 \mathrm{~K}$, while property degradation induced temperature increases are approximately $0.35 \mathrm{~K} /$ year at the present time (expected to decrease as the mission continues).

Figure 13 is a plot of temperature of a typical cold stage magic tee element over a 1 hour period. Maximum temperature variations of the order of $0.5 \mathrm{mK}$ peak to peak ( $<0.2 \mathrm{mK} \mathrm{rms}$ ) are observable, compared with the $0.5 \mathrm{mK}$ rms cyclic requirement during the spin period. Temperature variations correlated with the spin period cannot be observed on a one hour plot. However, after one month of data accumulation, the periodic temperature variation of a typical magic tee component over a spin period was determined to be $<7 \mu \mathrm{K}$.

Figures 14 and 15 are temperature plots of a primary reflector (top) and the V222 microwave magic tee over the period September 1, 2001 through October 1, 2001. The small discontinuity on September 14 was caused by a planned trajectory correction maneuver. The observatory angle to the sun remains $<22.5$ degrees throughout the TCM, preventing solar exposure to the cold stage, but the spin is stopped and the observatory is oriented correctly to perform the short thruster burn in the desired direction. The small temperature variation at that date is due to the thruster plume which is partly visible by the cold reflector and radiator. The temperature variation seen on both components on
September 24 was caused by a solar flare event. Solar flares result in energy deposition into nearly all spacecraft surfaces, regardless of orientation with respect to the sun. Small flares often resulted in temperature fluctuations of the order of $5-20 \mathrm{mK}$ at the top of the WMAP primary reflectors. The $70 \mathrm{mK}$ spike in

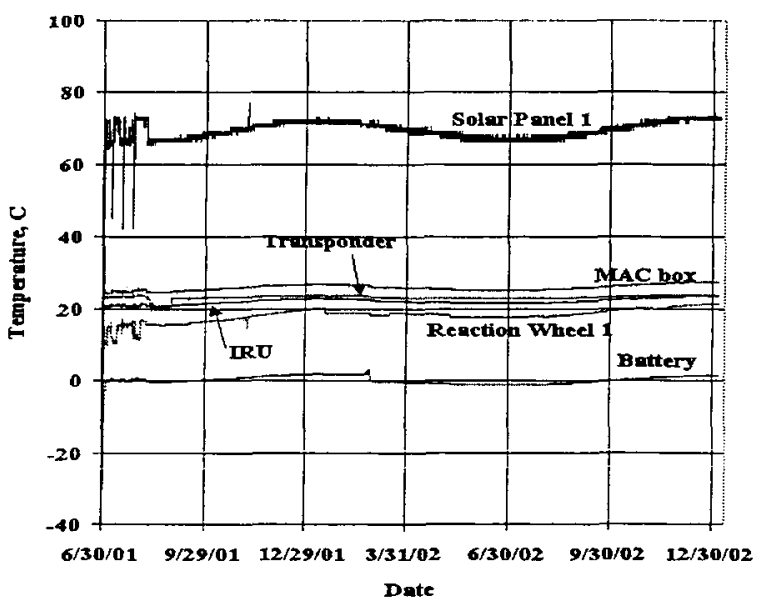

Figure 7. Selected WMAP Spacecraft Component Temperatures

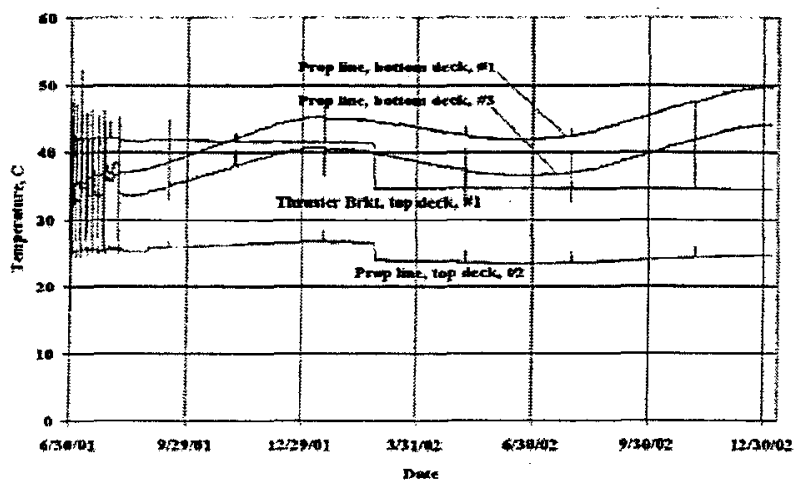

Figure 8. Selected WMAP Propulsion System Component Temperatures

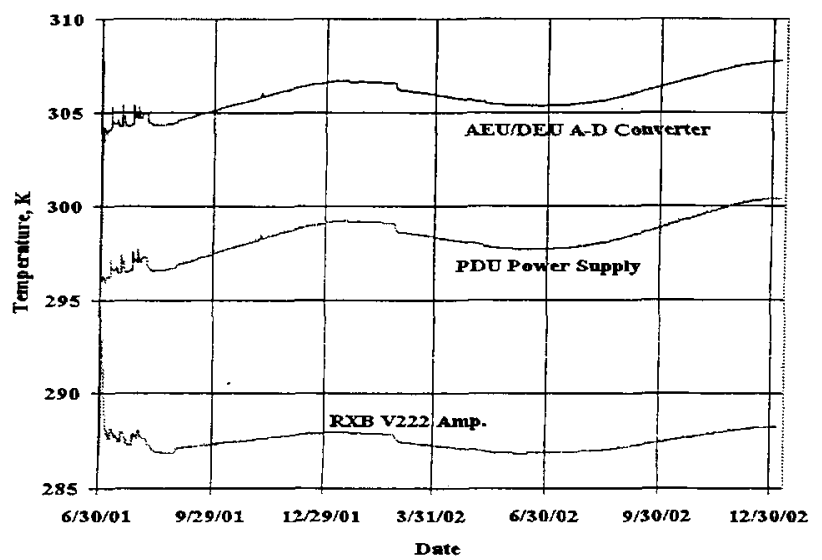

Figure 9. Selected Instrument Warm Stage Component Temperatures 


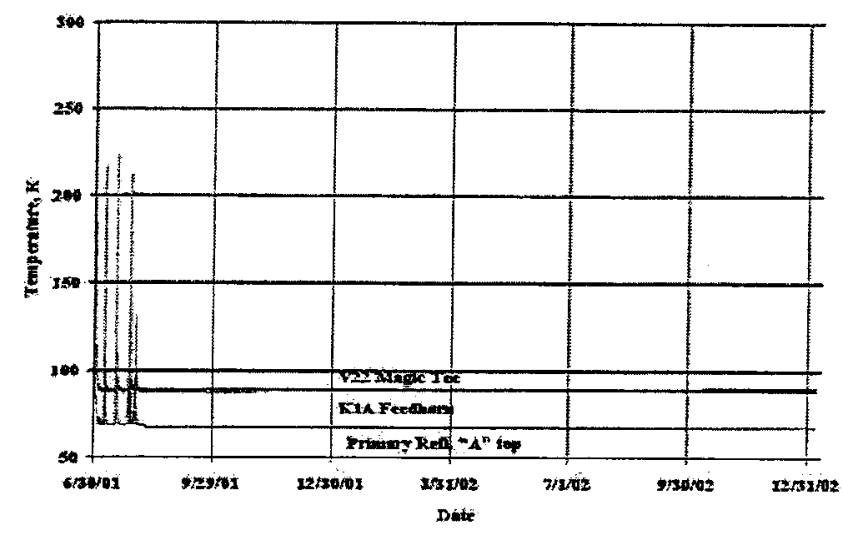

Figure 10. Selected Instrument Temperatures

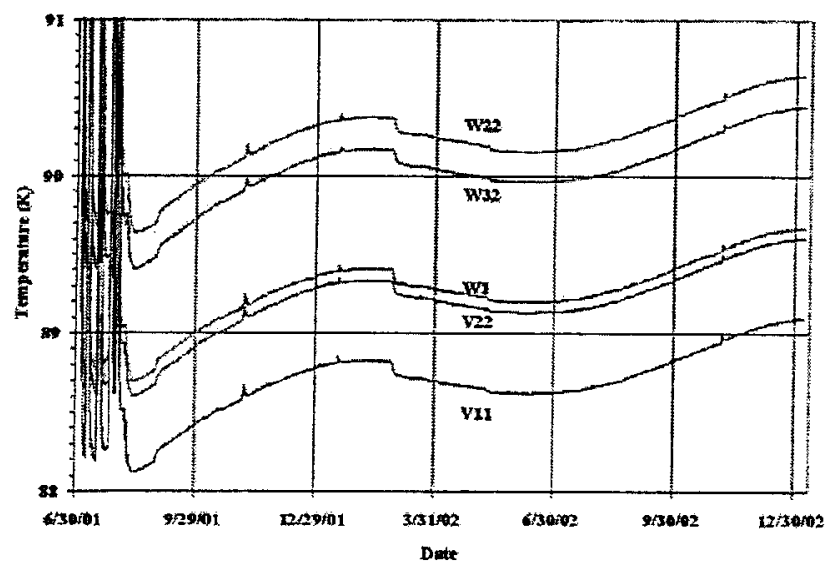

Figure 11. Cold Stage Magic Tee Temperatures While Observing

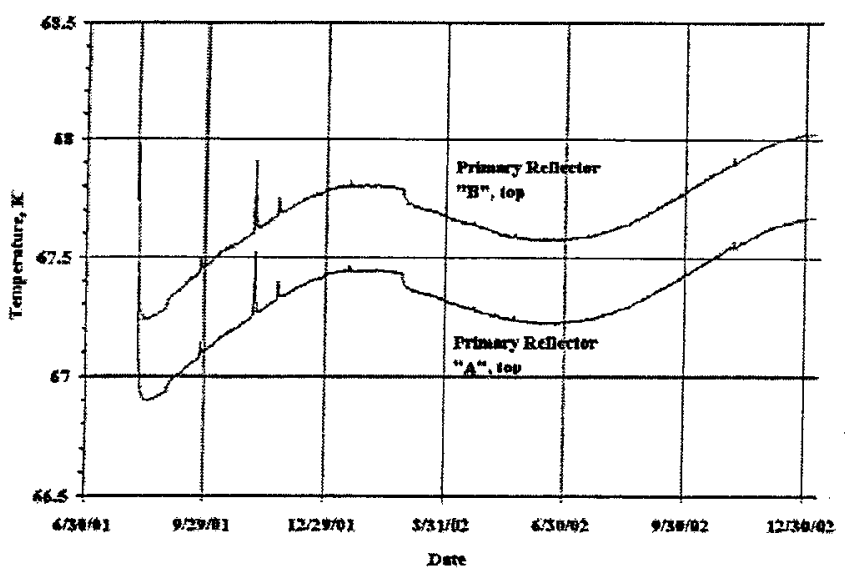

Figure 12. Reflector A,B Temperature (top) While Observing

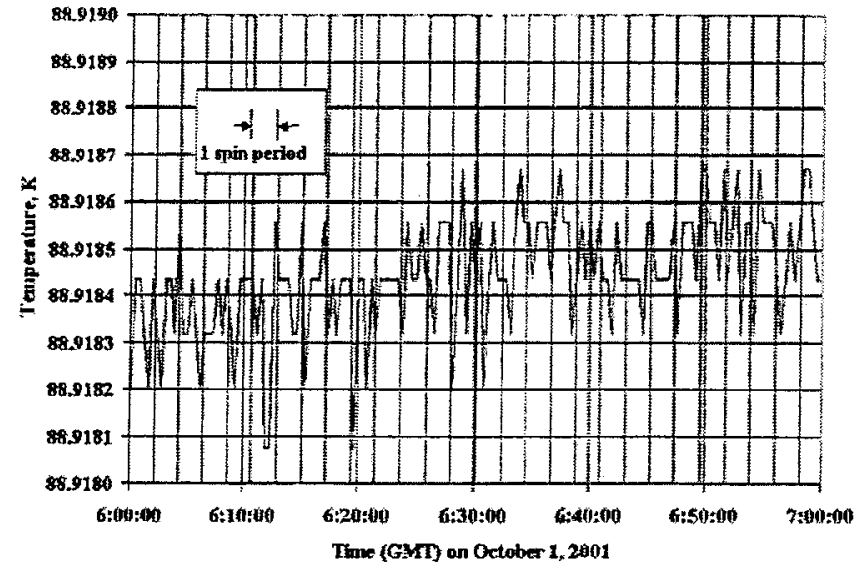

Figure 13. V222 Cold Magic Tee Temperature Variation over a Precession Period

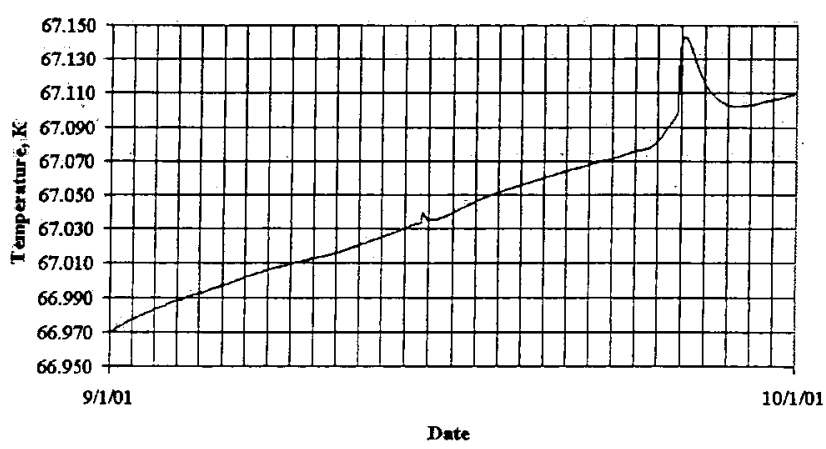

Figure 14. Effects of TCM, Solar Flare on Primary Reflector "A" (top) Temperature

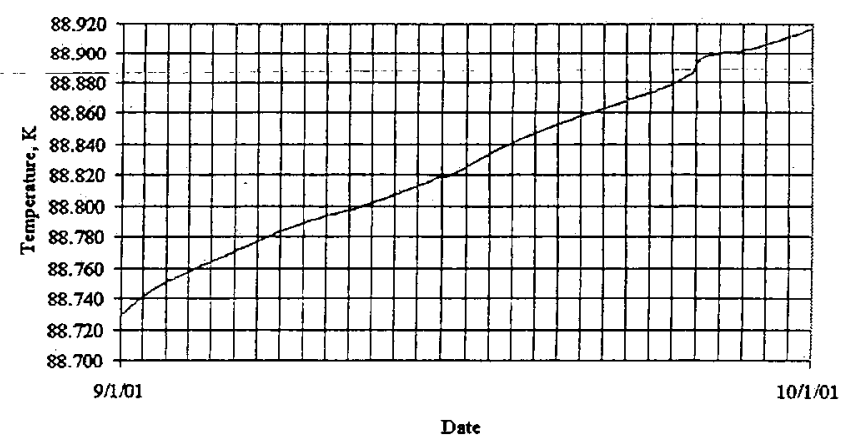

Figure 15. Effects of TCM, Solar Flare on V222 Magic Tee Temperature 
response to the event occurring on September 24, 2001 was one of the largest observed. The effects are substantially more pronounced at the top of the reflector than at the microwave element, which is more massive and situated in the interior of the instrument. The sensor on the top of the primary reflector was actually mounted on the back of the reflector's front facesheet, in a small area where the back facesheet and honeycomb were removed. The density and specific heat of the $25.4 \mathrm{~mm}$ thick facesheet at $70 \mathrm{~K}$ are approximately $0.0452 \mathrm{gm} / \mathrm{cm}^{2}$ and $0.215 \mathrm{~J} / \mathrm{gm}-\mathrm{K}$, respectively, yielding an area-based heat capacity of $0.0097 \mathrm{~J} / \mathrm{cm}^{2}-\mathrm{K}$ at the location of the sensor.

\section{CONCLUSION}

The WMAP observatory successfully meets all established thermal requirements in flight. Lessons learned from the mission may be applied to the design and testing philosophies of future missions that require passive cooling to achieve cryogenic temperatures with fine thermal stability, that will orbit the $L 2$ point, such as the James Webb Space Telescope. Extensive and early testing of high-fidelity mockup and ETU hardware is invaluable to mission success. Parasitic heat leaks resulting from workmanship and inherent design weaknesses could not have been found and fixed in the flight unit without early testing. Adequate thermal margins must be held as a design goal, and heat loads aggressively tracked against the margins throughout the design/testing phases. The thermal design of the instrument and observatory needed to meet steady state requirements should be determined early, particularly for those missions that maintain relatively constant solar exposure. Extensive analysis is required to investigate the transient performance during very brief solar exposures during the launch and early mission periods. Thermal performance during these brief periods may have a large impact on selection of long-lead coatings and materials.

\section{ACKNOWLEDGMENTS}

The efforts of many thermal engineers in the Thermal Engineering Branch at the NASA Goddard Space Flight Center, plus MLI blanket technicians at Mantech Corp., and Swales Corp., were required for the success of this mission. The authors wish to thank Mr. Dennis Hewitt, Thermal Engineering Branch Head during the project, for his support and leadership. The authors also wish to thank Dr. Gary Hinshaw and other members of the WMAP science team for their review and assistance in preparing this paper. We also appreciate the efforts of Mr. Mike Greason in providing the flight data for plotting, and Mr. Britt Griswold for providing several figures.

\section{ADDITIONAL SOURCES}

Additional information about the WMAP observatory, the mission, its scientific goals, and latest mission results may be found at the following official project website:

\section{http://map.gsfc.nasa.gov}

In addition, the following site provides $\mathrm{CMB}$ researchers with archive data from NASA missions, software tools, and links to other sites of interest:

\section{http://lambda.gsfc.nasa.gov/}




\section{APPENDIX}

Table A1. Thermal Requirements

A. Temperature Limits

Spacecraft Component

$\begin{array}{ll}\text { PDU } & \text { (warm instr. Box) } \\ \text { RXB } & \text { (warm instr. Box) }\end{array}$

AEU/DEU (warm instr. Box)

IRU

MAC

LMAC

PSE

VRAIL

Battery Cell

Star Trackers -CCD

Star Trackers -CCD Housing

Star Trackers - Bias Board.

Reaction Wheels

Reaction Wheel Elec.

Dig. Sun Sens. (DSS)

DSS Electronics

Transponders

Solar Array Panels

Propulsion Tank

Fuel Lines

Thruster Valves 1-2

Thruster Valves 3-8

Cold Stage Instrument

Component

Primary Reflector

Secondary Reflector

Instrument Truss

Cold DA's, Feeds, $\mu$ wave amps.

Gamma alumina cylinder

\section{Operating Operating Survival Survival Cold \\ Hot \\ Cold \\ Hot}

(C)

(C)

0

0

0

0

0

0

0

$-20$

$-10$

$-10$

$-10$

$-10$

0

0

$-20$

$-15$

$-10$

$-55$

10

10

10

10

\section{Operating Operating}

Cold

(K)

N/A

N/A

N/A

N/A

N/A

Hot
$(\mathrm{K})$
$<95$
$<95$
N/A
$<95$
N/A

(C)

$-10$

$-10$

$-10$

$-25$

$-10-50$

$-20 \cdot 50$

$-20 \quad 50$

$-30 \quad 50$

$-20 \quad 30$

$-20 \quad 53$

$-20 \quad 53$

$-20 \quad 52$

$-25 \quad 50$

$-25 \quad 50$

$-50 \quad 60$

$-25 \quad 45$

$-20 \quad 65$

$-65 \quad 103$

$5 \quad 55$

$5 \quad 60$

$5 \quad 125$

$5 \quad 125$

\section{B. Thermal Stability Requirements}

\section{Component}

AEU/DEU

PDU

FPA Cold Amps

RXB Electronics

Primary Reflectors

Secondary Reflectors

Feeds

OMTs

Cross Coupling wg.

Hybrid Tees

\section{Common Mode $\mathrm{mK} \mathrm{rms/spin} \mathrm{period}$}

3.6

3.6

0.5

0.5

10.0

10.0

2.2

2.0

0.7

0.7
Differential Mode

$\mathrm{mK} \mathrm{rms} / \mathrm{spin}$ period
N/A
N/A
N/A
N/A
2.0
2.0
0.13
0.13
0.04
0.04 
Table A2. Growth in Heat Loads (W) to WMAP Instrument Cold Stage Throughout the Project

$\begin{array}{lccccc}\text { Source } & \text { Initial Proposal } & \text { SCR } & \text { Peer Review } & \text { IDR } & \text { Pre-Launch } \\ & \mathbf{1 2 / 9 5} & \mathbf{7 / 9 6} & \mathbf{1 2 / 9 6} & \mathbf{3 / 9 7} & \mathbf{6 / 0 1} \\ \text { HEMT Dissipation } & 0.1 & 0.4 & 1.3 & 2.6 & 1.9 \\ \text { Conducted+Radiated, waveguide } & 1.5 & 1.8 & 2.8 & 2.8 & 2.2 \\ \text { Conducted, electrical wires } & 0.3 & 0.9 & 0.9 & 0.8 & 0.6 \\ \text { Conducted, ground strap } & 0.1 & 0.1 & 0.1 & 0.3 & 0.3 \\ \text { Conducted, supports } & 0.4 & 0.4 & 0.4 & 0.4 & 0.6 \\ \text { Conducted, RXB supports } & 0.9 & 0.9 & 0.2 & 0.5 & 0.6 \\ \text { Radiated, S/A, sunshade } & 0.7 & 0.7 & 0.8 & 0.8 & 1.7 \\ \text { Radiated, upper MLI } & 0.6 & 0.6 & 0.1 & 0.1 & 0.1 \\ \text { Radiated, RXB MLI } & - & - & - & 1.2 & 2.0 \\ \text { Radiated, other cold components. } & 1.4 & 1.4 & 1.0 & 1.0 & 1.0 \\ & & & & & 1.0\end{array}$


Table A3. Key System Tests, Results

$\begin{array}{lll}\text { Test } & \text { Date } & \text { Configuration } \\ \text { Reflector Evaluation } & 4 / 98 & \begin{array}{l}\text { Half of ETU TRS, in TV } \\ \text { chamber with LN2 shrouds }\end{array}\end{array}$

TRS Hot test

$10 / 99$

Flight TRS in TV chamber with LN2 shrouds, solar simulator used
2- $\quad$ New flight secondary $3 / 00$ reflectors (individually) in TV chamber with LN2 shrouds, solar simulator

Structural Thermal Mockup (STM) Parts 1,2,3

$\begin{array}{lll}\begin{array}{l}\text { New secondary } \\ \text { reflector tests }\end{array} & 2- & \begin{array}{l}\text { New flight secondary } \\ \text { reflectors (individually) in TV } \\ \text { chamber with LN2 shrouds, } \\ \text { solar simulator }\end{array} \\ 3 / 00\end{array}$

$5-$ $8 / 99$

High fidelity thermal mockup of RXB, microwave components, flight TRS used in Part 2, in TV chamber, Gasseous helium shroud walls
$1-$ $3 / 00$

$\begin{array}{ll}\text { Cold-Vibe-Cold } & 1- \\ \text { (CVC), Parts } 1 \text { and } 2 & 3 / 00\end{array}$

Flight instrument (cold stage and warm instrument boxes) were mounted on the flight gamma alumina cylinder, on a S/C mockup. Test took place in a TV chamber with LN2 walls
Observatory TV/TB $\quad 1-\quad$ Instrument section in flight Test, Parts 1,2,3 configuration in S/C, both in TV chamber. S/C exposed to LN2 shrouds, instrument cold stage in shrouds cooled with gaseous helium.

\section{Major Test Goals}

Qualify Reflector Design

(hot and cold), perform metrology measurements

Qualify reflector design under hot, cold conditions, apply thermal shock similar to flight

Qualify new coatings on secondary reflectors, expose them to thermal shock similar to flight

Measure parasitic heat leak at warm, cold I/F of $R X B$ and FPAS, determine steady state cold stage operating temperature
Detailed instrument performance tests, verify that $R X B$ thermal control was acceptable, collect thermal data to allow correlation of fight instrument thermal model

\section{Key Results}

Additional stiffening of primary reflectors needed for flight units. Design altered for flight reflectors.

Reflector redesign passed, but coatings on secondary reflectors later failed.

Secondary reflectors were remade, and coatings modified

Reflectors, coatings passed qualification

In Part 1, identified large parasitic heat leaks at RXBFPA I/F due to workmanship, inherent design fault. Problem investigated further in Part 2 , and the RXB-FPA I/F in the STM was fixed prior to part 3, to prove design adequacy for flight unit.

Boxes, FPA qualified, although bond line problems were discovered in specific HEMT amplifiers. RXB themal control was found to be inadequate in part 1. While HEMT amplifiers were being fixed for part 2, thermal control of $R X B$ was changed to include heat strap coupling to a thermal sink area. Rebuilt HEMT amps worked in part 2. RXB thermal design was found to be acceptable, but slightly overcooled. Sufficient data taken to enable correlation of thermal model of instrument.

Qualify thermal design of $\mathrm{S} / \mathrm{C}$ and instrument, determine shunt heater power required for prop. System components, correlate thermal models
Instrument and $S / C$ design was qualified. Adjustment to RXB thermal control heat straps, made after CVC test, were adequate. All other test goals met. 